\title{
OPÇÕES TÉCNICAS UTILIZADAS NO TRANSPLANTE PANCREÁTICO EM CENTROS BRASILEIROS
}

\section{TECHNICAL OPTIONS USED IN PANCREATIC TRANSPLANTS IN BRAZILIAN CENTERS}

\author{
Adriano Miziara Gonzalez,TCBC-SP'; Gaspar de Jesus Lopes Filho, TCBC-SP²; \\ Tarcísio Triviño, TCBC-SP ${ }^{3}$; Fabrízio Messetti ${ }^{4}$; Érica Bevilakua Rangel ${ }^{5}$; Claudio Melaragno ${ }^{5}$
}

\begin{abstract}
RESUMO: Objetivo: Analisar o perfil dos principais centros de transplantes do Brasil, quanto às opções técnicas no transplante de pâncreas. Método: Foi encaminhado um questionário por correio eletrônico (email) para um membro de cada equipe de 12 centros de transplante do Brasil, com casuística mínima de um transplante de pâncreas. O questionário continha 10 perguntas, abordando aspectos controversos e não padronizados. Resultados: A maioria dos centros $(90,9 \%)$ utiliza incisão mediana. O órgão de escolha a ser implantado primeiro foi principalmente o rim, em $63 \%$ dos centros. Em relação à drenagem venosa, 90,9\% utilizam a drenagem sistêmica. A ligadura da veia ilíaca interna é realizada em $54,5 \%$ dos centros. A maioria dos centros $(90,9 \%)$ utiliza a drenagem entérica para transplante combinado pâncreas-rim. Para o transplante de pâncreas isolado, apenas cinco centros responderam, sendo que dois utilizam a drenagem entérica e três a vesical. A utilização de dreno na cavidade abdominal ocorre em $63 \%$ dos centros. Em $72,7 \%$ dos centros é realizada algum tipo de indução na imunossupressão para o transplante combinado pâncreas-rim, sendo a imunossupressão básica a associação de tacrolimus (FK506), micofenolato mofetil (MMF) e corticóide. A antibioticoprofilaxia é realizada por todos os centros e profilaxia para fungos é realizada por seis centros $(54,5 \%)$. Oito centros $(72,7 \%)$ utilizam algum tipo de profilaxia para trombose vascular, em esquemas diversos. Conclusão: Existem diversos caminhos técnicos na condução do transplante pancreático. A falta de padronização dificulta a análise e a comparação dos resultados. Apesar dessa heterogeneidade das equipes, observamos uma tendência para a realização de incisão mediana, drenagem venosa sistêmica e exócrina entérica, com a utilização de algum tipo de profilaxia para trombose vascular nos transplantes combinados pâncreas-rim (Rev. Col. Bras. Cir. 2005; 32(1): 18-22).
\end{abstract}

Descritores: Transplante de pâncreas; Transplante/métodos; Transplante/estatística\& dados numéricos; Procedimentos cirúrgico operatório/métodos.

\section{INTRODUÇÃO}

Desde 1967, quando Kelly et al relataram as dificuldades no controle dos dois primeiros doentes transplantados de rim e pâncreas na Universidade de Minnesota, este procedimento enfrentou várias mudanças e hoje é uma terapêutica estabelecida ${ }^{1}$.

Em 1980, o Registro Internacional de Transplante de Pâncreas mostrava um índice de sobrevivência do enxerto pancreático de $21 \%$ e dos pacientes de $67 \%$, o que tornava os nefrologistas e endocrinologistas relutantes para o procedimento ${ }^{2}$. A melhora nas técnicas de imunossupressão, e de regimes de antibióticos, o entendimento dos eventos celulares associados à rejeição, a evolução das soluções de preservação, a evolução nas técnicas radiológicas com abordagens percutâneas e o aprimoramento nas técnicas cirúrgicas trouxeram resultados bastante animadores ${ }^{3}$.

Humar et al, em 2000, analisaram a incidência de complicações cirúrgicas observando uma diminuição importante na atualidade, que tem como possíveis fatores a diminuição da trombose vascular, entre outras, de $10,1 \%$ para $5,6 \%{ }^{4}$. Essas mudanças fizeram com que o Registro Internacional de
Transplante de Pâncreas demonstrasse, em 2001, sobrevivência do enxerto pancreático de $83 \%$ e dos pacientes de $95 \%$ para os transplantes combinados rim-pâncreas ${ }^{5}$.

Apesar da sensível melhora dos resultados, o transplante de pâncreas não tem uma padronização quanto à imunossupressão e à técnica cirúrgica. A análise de resultados é de difícil comparação pela heterogeneidade dos dados.

No Brasil, a experiência clínica com o TP ainda é inicial, com vários serviços em fase de implantação de progra$\mathrm{ma}^{6}$, mas, o número de transplantes de pâncreas vem crescendo. O objetivo desta pesquisa foi analisar o perfil dos principais centros de transplantes do Brasil, quanto às opções técnicas no transplante de pâncreas.

\section{MÉTODO}

Foi encaminhado um questionário por correio eletrônico(email) para um membro de cada equipe de 12 centros de transplante do Brasil, com casuística mínima de um transplante de pâncreas. O questionário continha 10 perguntas, abordando aspectos controversos e não padronizados, conforme mostra o Quadro I.

1. Médico Assistente e Coordenador do Programa de Transplante de Órgãos do Aparelho Digestivo da Disciplina de Gastroenterologia Cirúrgica do Hospital São Paulo - UNIFESP.

2. Professor Adjunto, Livre Docente da Disciplina de Gastroenterologia Cirúrgica do Hospital São Paulo - UNIFESP.

3. Professor Adjunto, Chefe do Programa de Transplante de Órgãos do Aparelho Digestivo da Disciplina de Gastroenterologia Cirúrgica do Hospital São Paulo - UNIFESP.

4. Médico Residente de Cirurgia Geral do Hospital São Paulo- UNFESP.

5. Médico Assistente da Disciplina de Nefrologia e do Programa de Transplante de Pâncreas -Rim do Hospital São Paulo- UNIFESP.

Recebido em 14/06/2004

Aceito para publicação em 29/09/2004

Trabalho realizado no Hospital São Paulo - UNIFESP. 


\section{RESULTADOS}

As respostas foram obtidas de onze equipes. Apenas um centro não respondeu ao questionário.

Quanto à primeira pergunta, abordando o tempo de espera, as respostas foram dadas com ressalvas e incompletas. Muitos não responderam por este ainda não ser um procedimento rotineiro.

Em relação à incisão utilizada, a maioria dos centros $(90,9 \%)$ utiliza incisão mediana e apenas um centro utiliza a incisão bilateral de "Gibson" nos transplantes combinados.

O órgão de escolha a ser implantado primeiro foi principalmente o rim, em $63 \%$ dos centros, em $27 \%$ o pâncreas e em um centro $(9 \%)$ não houve preferência.

Em relação à drenagem venosa, 90,9\% utilizam a drenagem sistêmica, mas um centro não definiu a preferência e utiliza os dois tipos de drenagem.

A ligadura da veia ilíaca interna é realizada em 54,5\% dos centros e 45,5\% não realizam esta manobra.

A maioria dos centros $(90,9 \%)$ utiliza a drenagem entérica para transplante combinado pâncreas-rim. Para o transplante de pâncreas isolado, apenas cinco centros responderam, sendo que dois utilizam a drenagem entérica e três a vesical.

A utilização de dreno na cavidade abdominal é feito na maioria dos centros (63\%), sendo que dois centros $(18 \%)$ não a utilizam e outros dois utilizam, dependendo do aspecto ao término da cirurgia. O tipo de dreno e o tempo de sua utilização não foram corretamente respondidos.

Em $72,7 \%$ dos centros é realizada algum tipo de indução na imunossupressão para o transplante combinado pâncreas-rim, sendo a imunossupressão básica a associação de tacrolimus (FK506), micofenolato mofetil (MMF) e corticóide.

A antibioticoprofilaxia é realizada por todos os centros em esquemas variados, quanto ao tipo do antibiótico e

1-Qual é o tempo médio de espera na fila para o doente ser transplantado?

2- Qual é a incisão geralmente utilizada?

3- Qual órgão é implantado primeiro: o rim ou o pâncreas? Esta preferência tem uma razão especial?

4- A drenagem venosa é sistêmica ou portal?

5- Se for sistêmica, a veia ilíaca interna é ligada rotineiramente? Se a fossa ilíaca direita estiver ocupada (ex.

Tx renal prévio) qual seria a outra opção?

6- Qual drenagem exócrina é utilizada: vesical ou entérica?

Se entérica, qual segmento intestinal é utilizado? Utiliza a técnica em "Y de Roux"?

7- Realiza a drenagem da cavidade? Se sim, que tipo de dreno e por quanto tempo?

8- Qual esquema de imunossupressão é utilizado?

9- Qual esquema de antibiótico profilático é utilizado e por quanto tempo?

10- Realiza profilaxia para trombose vascular? Qual?

Quadro I - Questionário enviado por correio eletrônico. tempo. Vale ressaltar que profilaxia para fungos é realizada por seis centros $(54,5 \%)$.

Oito centros $(72,7 \%)$ utilizam algum tipo de profilaxia para trombose vascular, em esquemas diversos. Em dois centros, utiliza-se apenas em determinadas situações e em um centro (9\%) não é utilizada nenhum esquema de profilaxia para trombose vascular.

\section{DISCUSSÃO}

No primeiro transplante de pâncreas-rim realizado em 1966, a técnica utilizada foi um segmento pancreático na fossa ilíaca e ligadura do ducto pancreático ${ }^{1}$. Como os resultados no início não eram satisfatórios, várias alternativas foram tentadas. Lillehei et al, em 1970, utilizaram todo o órgão pancreático com o duodeno e drenagem exócrina entérica ${ }^{7}$. Em 1973, Gliedman et al utilizaram o ureter para drenagem exócrina ${ }^{8}$. Em 1978, Dubernard et al obstruíram o ducto pancreático com polímero sintético ${ }^{9}$ e em 1980, Groth et al utilizaram drenagem entérica em Y de Roux ${ }^{10}$. O grupo de Minnesota relatou uma série de casos, deixando o ducto aberto dentro da cavidade ${ }^{11}$.

Várias técnicas foram utilizadas, mas foi a partir de 1982, quando Sollinger et al introduziram a drenagem vesical da secreção exócrina ${ }^{12}$, modificada por Nghiem e Corry ${ }^{13}$, que a técnica se estabeleceu e predominou nos EUA e Europa por quase duas décadas ${ }^{14}$.

Posteriormente, desde o final da década de 90 até os dias de hoje, o transplante de pâncreas passou por novas tendências, quanto à técnica e à manipulação clínica.

A incisão no transplante de pâncreas pode ser supra-inguinal bilateral ou mediana. Alguns autores consideraram como preferencial a incisão transversa bilateral, pela melhor exposição dos vasos ilíacos, menor íleo paralítico, menos dor e índices comparáveis de infecção ${ }^{15,16}$. No entanto, a preferência da maioria dos centros nacionais é pela incisão mediana $(90,9 \%)$. Esta tendência também é observada em centros internacionais ${ }^{17}$.

Apesar de o rim suportar por mais tempo a isquemia fria, no pâncreas esta só deve determinar maior índice de complicações quando ultrapassar 20 horas $^{18}$. Diminuir a incidência de necrose tubular aguda, realizar concomitantemente a cirurgia de mesa do pâncreas, evitar lesão pancreática pelos afastadores e diminuir edema pancreático foram as justificativas dadas para quem opta pela implantação renal primeiro. Quem opta por iniciar pelo pâncreas, alega ter este um menor tempo de isquemia além de poder observá-lo enquanto se realiza o transplante de rim.

A drenagem venosa sistêmica é uma técnica bem estabelecida, utilizada pela maioria dos centros e associada com excelente resultado a longo prazo ${ }^{1}$. Apresenta uma desvantagem teórica, pois levaria a um nível elevado periférico de insulina, podendo acarretar resistência da mesma e alteração no metabolismo lipídico ${ }^{19}$. A técnica de drenagem portal utilizada correntemente foi desenvolvida por Shokouh-Amiri ${ }^{20}$. Em 1995, Gaber et al utilizaram a drenagem venosa no sistema portal e exócrina no trânsito intestinal, observando menor incidência de complicações metabólicas e do trato urinário ${ }^{21}$. Stratta em 2003, apresentou experiência semelhante com dre- 
nagem venosa portal, com índices de sobrevivência do enxerto pancreático e do paciente em um ano de $82 \%$ e $97 \%$ respectivamente, sem aumento na incidência de trombose vascular $(7,5 \%)^{22}$

Em estudo retrospectivo, Philosophe et al observaram menor índice de rejeição com esta drenagem ${ }^{23}$. No Brasil, a maioria dos autores $(90,9 \%)$ utiliza a drenagem sistêmica, sendo que um centro não definiu a sua preferência e utilizou ambas.

O sistema vascular ilíaco direito é considerado preferencial para o implante pancreático. Apesar de alguns autores recomendarem a completa liberação da veia ilíaca direita ${ }^{24}$, tornando a anastomose venosa sem tensão e sem a necessidade de enxerto venoso, outros não a realizam e não relatam maiores índices de complicações ${ }^{25}$. Nesta pesquisa, a completa liberação da veia ilíaca interna é utilizada por $54,5 \%$ dos centros. A fossa ilíaca esquerda, região de maior dificuldade pela maior profundidade da veia e pela presença do sigmóide, é utilizada na indisponibilidade técnica da fossa ilíaca direita. Nesta situação, outra opção relatada é a drenagem portal. $\mathrm{Na}$ UNIFESP, foi utilizada a liberação da veia ilíaca direita nos primeiros 48 transplantes combinados pâncreas-rim. Na ausência da disponibilidade do lado direito, optou-se pela utilização da veia cava (ou confluência desta com a veia ilíaca comum direita). Esta técnica tornou o procedimento mais simples e rápido, sem a necessidade de ligadura de ramos venosos, tornando esta localização rotineira para o serviço.

Após a introdução da drenagem vesical para a secreção exócrina pancreática algumas complicações foram relatadas, tais como hematúria, cistites e uretrites, acidose metabólica, desidratação, fístulas urinárias, infecções urinárias e pancreatite de refluxo ${ }^{26-28}$.

Em 1997, Kuo et al compararam a drenagem vesical com a entérica, relatando menos infecção do trato urinário e complicações urológicas, com sobrevivência pancreática e dos doentes semelhantes ${ }^{3}$. Bloom et al reforçaram a tendência para a drenagem entérica, demonstrando menor número de complicações e tempo de internação, sem aumentar a perda de enxerto a longo prazo $^{29}$. Observações neste sentido foram mais freqüentes, culminando em inversão na preferência. Em 2001, após análise do Registro Internacional de Transplante de Pâncreas (IPTR), Gruessner e Sutherland demonstraram que $67 \%$ dos centros tem utilizado a drenagem entérica para transplante de pâncreas-rim ${ }^{5}$.

Para o transplante de pâncreas isolado, a análise do Registro Internacional demonstrou melhores resultados com drenagem vesical ${ }^{30}$, porém vale ressaltar que a drenagem entérica já foi realizada, sem comprometimento da sobrevivência do enxerto ${ }^{31}$.

No Brasil, a maioria dos centros $(90,9 \%)$ utiliza a drenagem entérica para transplante combinado pâncreas-rim. Esta predominância não se observa no transplante isolado de pâncreas. Nos cinco centros que responderam ao questionário, dois utilizam a drenagem entérica e três a vesical.

A introdução da ciclosporina em 1980 foi um marco na imunossupressão tendo contribuído sobremaneira para o desenvolvimento dos transplantes de órgãos sólidos. Num passado recente, a maioria dos transplantes simultâneos pâncreas-rim experimentavam pelo menos um episódio de rejeição celular aguda. A maioria dos centros passou a utilizar a associação de ciclosporina, azatioprina e corticóide, além da indução com anticorpos monoclonais ou policlonais $^{32}$. Com a introdução do tacrolimus (FK506) e micofenolato mofetil (MMF), observou-se redução nos índices de rejeição. Utilizando FK506, MMF e corticóide, sem indução, Reddy et al obtiveram índice de rejeição de $30 \%{ }^{33}$. Apesar dos resultados satisfatórios obtidos sem indução, a maioria dos centros apresentam resistência em eliminá-la. Esta tendência também foi observada neste questionário, onde $72,7 \%$ dos centros utilizam algum tipo de indução na imunossupressão para o transplante combinado pâncreasrim. Na UNIFESP, para o transplante combinado pâncreas-rim, é utilizada a associação de FK506, MMF e corticóide, sem indução, e se observa rejeição aguda em $21,5 \%$ dos doentes.

Quanto maior a imunossupressão utilizada, maior o risco de infecção. Esta parece ser a grande responsável pelos óbitos nos transplantes combinados pâncreas-rim em nosso meio $^{34}$. A profilaxia com antibióticos para o transplante de pâncreas é universal, porém, como em todos os transplantes, o risco infeccioso não é só bacteriano, mas também viral e fúngico. Casos de ruptura vascular por infecção fúngica já foram descri$\operatorname{tos}^{35}$. Na UNIFESP, não se utiliza profilaxia para fungos e, em 72 transplantados pâncreas-rim, houve necessidade de tratamento fúngico, mesmo que empiricamente, em 11,4\%, sem nenhum óbito secundário a fungos. Neste questionário nacional, a profilaxia para fungos é realizada em $54,5 \%$ dos centros.

As tromboses vasculares normalmente acarretam a perda do enxerto e representam a principal causa de perda pancreática não imunogênica após o transplante pancreáti$\mathrm{co}^{36}$. Kandaswamy et al, em 1999, observaram redução significativa na incidência de trombose vascular de $11,2 \%$ para $6,1 \%$, com o uso de FK506 e anticoagulantes, apesar do aumento na incidência de sangramento no período pós operatório ${ }^{37}$. Apesar da maioria dos centros utilizarem profilaxia para trombose vascular (inclusive neste questionário nacional, onde apenas um centro não a utiliza) vale ressaltar que esta não é uma conduta universal. Sollinger et al, em 1998, apresentaram casuística de 500 transplantes simultâneos pâncreas-rim, sem utilização de anticoagulantes e com incidência de trombose vascular de $1 \%{ }^{2}$.

Podemos concluir que existem diversos caminhos técnicos na condução do transplante pancreático. A falta de padronização dificulta a análise e a comparação dos resultados. Apesar dessa heterogeneidade das equipes, observamos uma tendência para a realização de incisão mediana, drenagem venosa sistêmica e exócrina entérica, com a utilização de algum tipo de profilaxia para trombose vascular nos transplantes combinados pâncreas-rim.

\section{AGRADECIMENTOS}

Adriano Miziara Gonzalez - Hospital São Paulo - Escola Paulista de Medicina - São Paulo, SP

Álvaro Antônio Bandeira Ferraz - Hospital das Clínicas da Universidade Federal de Pernambuco - Recife, PE

André de Moricz - Hospital Santa Casa de Misericordia de São Paulo - São Paulo, SP 
Ilka de Fátima S. F. Boin - Hospital das Clinicas - UNICAMP Campinas, SP

João Eduardo Leal Nicoluzzi - Hospital Maternidade Angelina Caron - Campina Grande, PR

José Marcus Raso Eulálio - Universidade Federal do Rio de Janeiro - Rio de Janeiro, RJ

José Maria Gross Figueiró - Hospital Felício Rocha - Belo Horizonte, $\mathrm{MG}$
Marcelo Perosa de Miranda - Hospitais São Camilo, Beneficência Portuguesa e Albert Einstein - São Paulo, SP

Roberto Ferreira Meirelles Jr - Hospital de Base - FUNFARME - São José do Rio Preto, SP

Telésforo Bacchella - Hospital das Clínicas de São Paulo FMUSP - São Paulo, SP

Salvador Gullo Neto - Hospital São Lucas da PUC - Porto Alegre, RS

\begin{abstract}
Background: To analyze the profile of the most important Brazilian Transplant Centers regarding technical options in the pancreas transplant. Methods: A query was sent by electronic mail for the 12 Brazilian Transplant Centers with at least one pancreas transplant performed. The query included ten questions approaching controversial and non-standard technical aspects. Results: Midline abdominal incision is used in $90.9 \%$ of the Centers. The first organ to be implanted is the kidney in $63 \%$ of the Centers. Regarding the venous drainage, $90.9 \%$ perform systemic drainage. In $54.5 \%$ of the Centers the internal iliac vein is ligated. For combined pancreas-kidney transplant $90.9 \%$ of the teams perform enteric drainage. Five Centers answered about isolated pancreas transplant, two of them use enteric drainage and the other three prefer to utilize the bladder. $63 \%$ of the surgical teams use abdominal drain. $72.7 \%$ of the Centers adopt immunosupression induction for the combined pancreas-kidney transplant. The basic immunosuppression was an association between tacrolimus (FK506), and mofetil microfenolato $(M M F)$, and corticoids. While antibiotic prophylaxis is performed in all the 12 Centers, fungus prophylaxis is routinely made in six of them. Eight Centers (72.7\%) adopt vascular thrombosis prophylaxis by several different protocols.Conclusion: There are various technical medical protocols on how to conduct a pancreas transplant patient. The lack of homogeneity in the protocols makes it more difficult to analyze and compare the results. Nevertheless we can conclude that in combined pancreas-kidney transplant there is a preference towards midline abdominal incision, and vein systemic and enteric drainage, and vascular thrombosis prophylaxis.
\end{abstract}

Key words: Pancreas transplantation; Transplantation/methods; Transplantation/statistics \& numerical data; Surgical procedures, operative/methods.

\section{REFERÊNCIAS}

1. Kelly WD, Lillehei RC, Merkel FK, et al. - Allotransplantation of the pancreas and duodenum along with the kidney in diabetic nephropathy. Surgery, 1967,61(6):827-837.

2. Sollinger HW, Odorico JS, Knechtle SJ, et al. - Experience with 500 simultaneous pancreas-kidney transplants. Ann Surg, 1998,228(3):284-296.

3. Kuo PC, Johnson LB, Schweitzer EJ, et al. - Simultaneous pancreas/kidney transplantation - a comparison of enteric and bladder drainage of exocrine pancreatic secretions. Transplantation, 1997,63(2):238-243.

4. Humar A, Kandaswamy R, Granger D, et al. - Decreased surgical risks of pancreas transplantation in the modern era. Ann Surg, 2000,231(2):269-275.

5. Gruessner AC, Sutherland DE - Analysis of United States (US) and non-US pancreas transplants reported to the United network for organ sharing (UNOS) and the international pancreas transplant registry (IPTR) as of October 2001. Clin Transpl, 2001, 41-72

6. Perosa M, Genzini T, Neto SG, et al. - Transplante de pâncreas com órgãos de doadores limítrofes. J Bras Transpl, 2002; Vol $5: 1$

7. Lillehei RC, Simmons RL, Najarian JS, et al. - Pancreaticoduodenal allotransplantation: experimental and clinical experience. Ann Surg, 1970,172(3):405-436.

8. Gliedman ML, Gold M, Whittaker J, et al. - Clinical segmental pancreatic transplantation with ureter-pancreatic duct anastomosis for exocrine drainage. Surgery, 1973,74(2):171-180.

9. Dubernard JM, Traeger J, Neyra P, et al. - A new method of preparation of segmental pancreatic grafts for transplantation: trials in dogs and in man. Surgery,1978,84(5):633-639.
10. Groth CG, Lundgren G, Ostman J, et al. - Experience with nine segmental pancreatic transplantations in preuremic diabetic patients in Stockholm. Transplant Proc,1980,12(4 Suppl 2):68-71.

11. Sutherland DE, Goetz FC, Najarian JS - One hundred pancreas transplants at a single institution. Ann Surg, 1984,200(4):414-40.

12. Cook K, Sollinger HW, Warner T, et al. - Pancreaticocystostomy: an alternative method for exocrine drainage of segmental pancreatic allografts. Transplantation,1983,35(6):634-636.

13. Nghiem DD, Corry RJ - Technique of simultaneous renal pancreatoduodenal transplantation with urinary drainage of pancreatic secretion. Am J Surg, 1987,153(4):405-406.

14. Gruessner A, Sutherland DE - Pancreas transplantation in the United States (US) and non-US as reported to the United Network for Organ Sharing (UNOS) and the International Pancreas Transplant Registry (IPTR). Clin Transpl, 1996,47-67.

15. Barone GW, Sailors DM, Ketel BL - Combined kidney and pancreas transplants through lower transverse abdominal incisions. Clin Transplant,1996,10(3):316-319.

16. Douzdjian V, Gugliuzza KK - The impact of midline versus transverse incisions on wound complications and outcome in simultaneous pancreas-kidney transplants: a retrospective analysis. Transpl Int, 1996,9(1):62-67.

17. Di Carlo V, Castoldi R, Cristallo M, et al. - Techniques of pancreas transplantation through the world: an IPITA Center survey. Transplant Proc, 1998,30(2):231-241.

18. Humar A, Kandaswamy R, Drangstveit MB, et al. - Prolonged preservation increases surgical complications after pancreas transplants. Surgery, 2000,127(5):545-551.

19. Hughes TA, Gaber AO, Amiri HS, et al. - Kidney-pancreas transplantation. The effect of portal versus systemic venous drainage of the pancreas on the lipoprotein composition. Transplantation, 1995,60(12):1406-1412. 
20. Shokouh-Amiri MH, Gaber AO, Gaber LW, et al. - Pancreas transplantation with portal venous drainage and enteric exocrine diversion: a new technique. Transplant Proc, 1992,24(3):776777.

21. Gaber AO, Shokouh-Amiri MH, Hathaway DK, et al. - Results of pancreas transplantation with portal venous and enteric drainage. Ann Surg, 1995,221(6):613-624.

22. Stratta RJ, Shokouh-Amiri MH, Egidi MF, et al. - Long-term experience with simultaneous kidney-pancreas transplantation with portal-enteric drainage and tacrolimus/mycophenolate mofetil-based immunosuppression. Clin Transplant, 2003,17(Suppl 9):69-77.

23. Philosophe B, Farney AC, Schweitzer EJ, et al. - Superiority of portal venous drainage over systemic venous drainage in pancreas transplantation: a retrospective study. Ann Surg, 2001,234(5):689-696.

24. Schweitzer EJ, Bartlett ST - Wound complications after pancreatic transplantation through a kidney transplant incision. Transplant Proc, 1994,26(2):461.

25. Hopt UT, Busing M, Schareck W, et al. -Prevention of early postoperative graft thrombosis in pancreatic transplantation. Transplant Proc,1993,25(4):2607-2608.

26. Ciancio G, Burke GW, Nery JR, et al. - Urethritis/dysuria after simultaneous pancreas-kidney transplantation. Clin Transplant, 1996,10(1 Pt 1):67-70.

27. Ciancio G, Burke GW, Nery J, et al. - Urological complications following simultaneous pancreas-kidney transplantation Transplant Proc,1995,27(6):3125-3126.

28. Ciancio G, Burke GW, Roth D, et al. - Reflux pancreatitis after simultaneous pancreas-kidney transplantation treated by alpha 1 blocker. Transplantation, 1995,60(7):760-761.

29. Bloom RD, Olivares M, Rehman L, et al. - Long-term pancreas allograft outcome in simultaneous pancreas-kidney transplantation: a comparison of enteric and bladder drainage. Transplantation, 1997,64(12):1689-1695.
30. Gruessner AC, Sutherland DE, Gruessner RW. - Enteric versus bladder drainage for solitary pancreas transplants- a registry report. Transplant Proc,2001,33(1-2):1678-1680.

31. Odorico JS, Pirsch JD, Becker YT, et al. - Results of solitary pancreas transplantation with enteric drainage: is there a benefit from monitoring urinary amylase levels? Transplant Proc,2001,33(1-2):1700.

32. Bruce DS, Sollinger HW, Humar A, et al. - Multicenter survey of daclizumab induction in simultaneous kidney-pancreas transplant recipients. Transplantation,2001,72(10):1637-1643.

33. Reddy KS, Stratta RJ, Shokouh-Amiri H, et al. - Simultaneous kidney-pancreas transplantation without antilymphocyte induction. Transplantation,2000,69(1):49-54.

34. Nicoluzzi JEL, Marmanillo CGWC, Repka JCD, et al. - Resultados do transplante pancreático em um centro brasileiro. Rev Col Bras Cir,2003,30(6): 436-441.

35. Ciancio G, Burke GW, Viciana AL, et al. - Destructive allograft fungal arteritis following simultaneous pancreas-kidney transplantation. Transplantation, 1996,61(8):1172-1175.

36. Gilabert R, Fernandez-Cruz L, Real MI, et al. - Treatment and outcome of pancreatic venous graft thrombosis after kidneypancreas transplantation. Br J Surg,2002,89(3):355-360.

37. Kandaswamy R, Humar A, Gruessner AC, et al. - Vascular graft thrombosis after pancreas transplantation: comparison of the FK 506 and cyclosporine eras. Transplant Proc,1999,31 (1-2):602-603.

Endereço para correspondência:

Fabrizio Messetti

Rua Borges Lagoa,836/64

Vila Clementino

04038-001 - São Paulo - SP 